Understanding, trusting, and applying scientific insights to improve your health: A latent profile analysis approach

\author{
Nejc Plohl ${ }^{1}$ and Bojan Musil ${ }^{1}$ \\ ${ }^{1}$ Department of Psychology, University of Maribor, Slovenia
}

Word count $=7745$ (including the abstract, tables, references, figure captions, and the appendix)

Correspondence concerning this article should be addressed to Nejc Plohl, Department of Psychology, University of Maribor, Koroska cesta 160, 2000 Maribor, Slovenia. Email: nejc.plohl1@um.si 


\begin{abstract}
Various leading causes of death can be prevented or delayed through informed decision-making and lifestyle changes. Previous work has, to some extent, linked such health-promoting behavior (HPB) with variables capturing individuals' understanding of science, trust in science, and capacity to obtain, process, and apply evidence-based information in the health context. However, empirical research on the relationship between scientific knowledge, trust in science, health literacy, and HPB is scarce. Additionally, no study has investigated whether these characteristics interact to form homogeneous, highrisk subgroups of the population. The present online study $(N=705)$ revealed that trust in science and health literacy were positively related to a wide array of HPB (e.g., healthy nutrition, physical activity, stress management), while scientific knowledge was only positively associated with COVID-19 vaccination intention. Furthermore, the results of latent profile analyses yielded four subgroups (i.e., low, moderate, and high levels of all three variables and a varied profile exhibiting very low trust in science, low health literacy, and moderate scientific knowledge). The identified subgroups differ significantly in their HPB and other variables of interest. Hence, the present study offers some guidance on which groups may be targeted with public health campaigns and how they may be designed.
\end{abstract}

Keywords: scientific knowledge, trust in science, health literacy, health, COVID-19, latent profile analysis 


\section{Understanding, trusting, and applying scientific insights to improve your health: A latent profile analysis approach}

\section{Introduction}

There is a growing body of literature claiming that many of the world's leading causes of death are preventable. A classic example illustrating this point is coronary heart disease (CHD), responsible for about 9 million deaths worldwide in 2019, substantially more than the approximately 7 million deaths two decades ago (World Health Organization, 2020). Even when such statistics are adjusted for temporal changes in the age of the population, CHD is still the number one cause of mortality, killing about 83 Americans every hour (Centers for Disease Control and Prevention, 2012). According to meta-analyses focusing on CHD prevention, a significant proportion of these deaths could have been prevented or delayed by various health-promoting behaviors (HPB) such as physical activity (Berlin \& Colditz, 1990; Rhodes et al., 2017; Sattelmair et al., 2011), healthy diet (Dauchet et al., 2006; Law et al., 1994), and improved stress management (Kivimaki et al., 2006; Richardson et al., 2012). Naturally, CHD is not the only major cause of death that could be reduced through improved decision-making and lifestyle changes; some cancers are also preventable through public health interventions (Bode \& Dong, 2009), as is COVID-19, mainly due to recent developments in understanding how the disease is spread and how the public can be inoculated against the virus (Kim et al., 2021; Lotfi et al., 2020). However, it is far from given that public health interventions aimed at promoting beneficial behaviors and curbing these diseases are indeed successful in influencing lifestyle choices and informing health decisions. For example, the prevalence of obesity in the United States continues to rise sharply despite numerous attempts to address this problem (Hales et al., 2020). Similarly, recent studies conducted on large samples show that approximately a quarter of US citizens do not plan to get vaccinated against COVID-19 despite various public health campaigns highlighting the safety and efficacy of available vaccines (Khubchandani et al., 2021). 
Although decisions related to health, such as the decision to be vaccinated, are notoriously complex and influenced by many factors (MacDonald \& the SAGE Working Group on Vaccine Hesitancy, 2015), the present paper argues that people are less likely to benefit from public health campaigns and adopt behaviors that would benefit their health: 1) if they do not understand the general science (or evidence) on which they are based, 2) do not trust the primary source of evidence (i.e., scientists), 3) and have difficulties obtaining, processing, and applying health information specifically.

The first factor, scientific knowledge or scientific literacy (1), is often regarded as a synonym with public understanding of science and generally includes components such as knowledge of the substantive content of science and the ability to distinguish it from non-science (Holbrook \& Rannikkmae, 2009; Laugksch, 2000). Although empirical studies of its relationship with HPB are scarce, often indirect, and inconclusive (Allum et al., 2014; Hornsey et al., 2021), improved decision-making in the context of health is hypothesized to be an important micro-level benefit of high scientific literacy, as decisions in this domain often demand some understanding of science and its processes (Laugksch, 2000; Yacoubian, 2018). The second factor, trust in science (2), refers to the extent to which individuals believe that scientific claims are honest and accurate reflections of researchers' work (Committee on Science, Engineering, 2009). While this variable has traditionally been used as an outcome, not as a predictor, recent studies related to COVID-19 identified trust in science as one of the critical variables that determine individuals' compliance with COVID-19 prevention guidelines (Pagliaro et al., 2021; Plohl \& Musil, 2021). The third factor, health literacy (3), is set explicitly in the health context (not science in general) and should not be equated with scientific literacy. Instead, it generally includes aspects such as basic health knowledge, applying this knowledge to make health decisions, as well as skills and motivation to find, use and assess the validity of health information (Ploomipuu et al., 2019). Higher health literacy is consistently associated with better health outcomes, including higher indulgence in HPB, and is thus considered as one of the critical public health goals (Berkman et al., 2011; Nutbeam, 2000; Von Wagner et al., 2007). 
Although all three factors described above could hence play an essential role in determining health decisions and lifestyle, the existing body of knowledge has several shortcomings. First, only a few empirical studies have actually investigated to what extent scientific knowledge and trust in science are individually related to a wide array of HPB. Second, due to the lack of studies exploring all three factors simultaneously, in a single study, it remains relatively unclear whether they exhibit similar patterns of correlations with HPB (and other variables of interest), how they compare in terms of the strength of association with HPB, as well as how they relate to each other. Third, though the variables are theoretically related yet distinct, it is not clear if (or how) they interact to form subgroups of individuals who are at exceptionally high risk in terms of their health outcomes.

In the present study, we employ a person-centered approach (as opposed to variable-centered approaches) to analysis, specifically latent profile analysis (LPA), to identify complex patterns of relationships between scientific knowledge, trust in science, and self-reported health literacy. Once such subgroups are identified, they will be compared in terms of their health-related outcomes (i.e., healthpromoting lifestyle including nutrition, physical activity, stress management, and health responsibility, compliance with COVID-19 prevention guidelines, and COVID-19 vaccination intention). Lastly, we will compare subgroups based on different variables traditionally treated as predictors of indicators used, such as education level, religiosity, political conservatism, and conspiracy ideation (e.g., Hornsey et al., 2021; Rutjens et al., 2018; Rutjens \& Lee, 2020), and determine the key predictors of profile membership. This knowledge could help us unravel at-risk groups that may be particularly addressed with public health campaigns in the future. Furthermore, this study's results could provide critical knowledge on how such campaigns should be designed to overcome the main barriers to the adoption of HPB in these groups. 


\section{Methods}

\subsection{Participants}

A total of 1066 participants from the United States started to fill out the survey. After excluding participants who dropped out $(N=143)$ and participants who were not attentive enough (i.e., failed two or all three attention checks; $N=218$ ), the final sample included 705 participants. Slightly less than twothirds of the sample was female (61.0\%), followed by participants identifying as male (37.9\%) or nonbinary $(1.1 \%)$. The age of participants ranged from 18 to 93 years, with a mean of 44.92 years $(S D=$ 17.80). In terms of their educational level, most respondents had a higher secondary education (51.8\%), followed by those with a bachelor's degree $(24.1 \%)$, a master's degree (8.9\%), primary education or less (7.5\%), lower secondary education (6.0\%), and a doctoral degree (1.7\%). Additionally, the majority of participants had annual incomes of \$25,000-49,999 (26.8\%), \$10,000-24,999 (21.6\%), or \$50,000-74,999 $(15.9 \%)$.

\subsection{Measures}

\subsubsection{Scientific knowledge}

The apprehension of basic scientific facts was measured using 11 objective questions (e.g., "Electrons are smaller than atoms") drawn from previous studies examining general scientific knowledge that is not particularly religiously contested (Impey et al., 2011; Johnson et al., 2015; McPhetres \& Zuckerman, 2018). Participants were able to answer with "True", "I don't know" or "False" and their responses were scored such that correct answers were assigned one point and false or "I don't know" answers were assigned zero points. The short objective test had satisfactory internal consistency $(\alpha=.68)$.

\subsubsection{Trust in science}

Trust in science was measured with the Trust in Science and Scientists Inventory, which captures a general level of trust in science and scientists (Nadelson et al., 2014). While the scale originally consists 
of 21 items answered on a 5-point agreement scale ranging from one ("Strongly disagree") to five ("Strongly agree"), we used a shorter version with 14 items that has previously exhibited an acceptable measurement model and good internal consistency (Plohl \& Musil, 2021). This was also the case in the present study $(\alpha=.92)$. Example item: "Scientific theories are trustworthy".

\subsubsection{Health literacy}

Individuals' self-reported knowledge, motivation, and competencies to access, understand, appraise and apply health information were measured using the short form of the European Health Literacy Survey Questionnaire, which consists of 16 items (HLS-EU-Q16; Pelikan et al., 2019), such as "How easy is it for you to understand information in the media on how to get healthier?". These items were answered on a 5-point scale ranging from "Very difficult" to "Very easy". The scale exhibited great internal consistency $(\alpha=.92)$.

\subsubsection{Conspiracy ideation}

The 15-item Generic Conspiracist Beliefs scale was used to measure individual differences in generic conspiracist ideation (Brotherton et al., 2013). The scale includes subscales on government malfeasance, extraterrestrial cover-up, malevolent global conspiracies, personal wellbeing, and control of information, which are answered on a 5-point scale ranging from "Definitely not true" to "Definitely true". As in previous studies (e.g., Brotherton et al., 2013), these subscales were combined into the general second-order factor (e.g., "The spread of certain viruses and/or diseases is the result of the deliberate, concealed efforts of some organization"). The scale showed great internal consistency ( $\alpha=$ $.95)$.

\subsubsection{Health-promoting lifestyle}

To measure the extent to which adults engage in a health-promoting lifestyle, we used the HealthPromoting Lifestyle Profile II (HPLP II; Walker \& Hill-Polerecky, 1996). While the scale originally contains 52 items and six subscales, we only used four of them in the present study, namely: nutrition (9 
items, e.g., "Choose a diet low in fat, saturated fat, and cholesterol"; $\alpha=.82$ ), physical activity (8 items, e.g., "Take part in leisure-time (recreational) physical activities (such as swimming, dancing, bicycling)"; $\alpha=.88$ ), health responsibility (9 items, e.g., "Report any unusual signs or symptoms to a physician or other health professional"; $\alpha=.83$ ), and stress management (8 items, e.g., "Get enough sleep"; $\alpha=.81$ ). The subscales can also be combined into a total health-promoting lifestyle score $(\alpha=.94)$. Answers to all the items are given on a frequency scale from 1 ("Never") to 4 ("Routinely").

\subsubsection{COVID-19 compliance}

Compliance with COVID-19 prevention guidelines was measured using an 11-item scale, which tackles different COVID-19 preventive behaviors outlined by the World Health Organization, Centers for Disease Control and Prevention, and European Center for Disease Prevention and Control (e.g., "Frequently washing your hands with soap and water for at least 20 seconds"; Plohl \& Musil, 2021). Participants were asked to what extent they act per these guidelines, to which they responded using a 4point frequency scale from "Not at all" to "To a great extent". The scale exhibited great internal consistency $(\alpha=.90)$.

\subsubsection{COVID-19 vaccination intention}

We also asked participants whether they have already received the COVID-19 vaccine ("Yes" or "No"). Those who answered with "No", received one additional question aimed at measuring their COVID-19 vaccination intention. The response format for this question was a 5-point agreement scale ranging from "Definitely not" to "Definitely yes".

\subsubsection{Socio-demographic data}

Various socio-demographic data were collected during the study (gender, age, education level, annual income, religiosity, and political conservatism). Education level was assessed by asking participants to mark their highest level of education, with response options ranging from "Primary education or less" to "Doctoral degree or equivalent". Religiosity was assessed with one item; 
specifically, participants were asked to express their level of agreement with the statement "I am very religious" on a 7-point agreement scale from "Strongly disagree" to "Strongly agree". Finally, political conservatism was measured using the following two questions: "How would you describe your political outlook with regard to (1) social /(2) economic issues?", answered on a response scale from 1 (“Very liberal") to 7 ("Very conservative"; e.g., Talhelm et al., 2015). Since the questions were highly correlated, they were treated as two indicators of political conservatism $(\alpha=.94)$.

\subsubsection{Attention checks}

Three attention checks were inserted into the survey, approximately every 1-2 pages. Specifically, we used the directed questions, which tell the participants to give specific answers (e.g., "This is a control question. Mark “Agree” and move on”; Maniaci \& Rogge, 2014). Attention checks were scored by summing the number of mistakes each subject made on these items to create scores ranging from 0 to 3.

\subsection{Procedure}

Participants were recruited using Prime Panels, a compilation of online research panels. According to previous examinations, Prime Panels generally provide diverse participants (in terms of their age, religiosity, education, and political attitudes) who are less used to classic protocols compared to some other panel providers (Chandler et al., 2019). Anyone aged 18 years or above was eligible to participate in the online study. While no specific sample size calculations were performed before the study, we aimed to surpass the median sample size observed in past research $(N=494$; Spurk et al., 2020). We also considered a general rule of thumb that a minimum sample size of about 500 participants is needed to accurately identify the latent profiles (Nylund et al., 2007).

Participants were first given a brief description of the study, including basic information about the study's objectives and methodology, and were informed that their participation was anonymous, voluntary, and could be terminated at any time. After consenting to participate in the study, participants were asked to fill out the questionnaire battery described earlier. For individuals who decided to 
participate in the study, the study procedure took approximately 10-15 minutes to complete. The study was conducted in accordance with the Declaration of Helsinki. Ethical review and approval were not required for this study according to the national and institutional guidelines.

\subsection{Statistical analyses}

Statistical analyses were performed using IBM SPSS Statistics 26.0 and Mplus 8.0. In the first step, participants who dropped out before the end of the study and inattentive participants were excluded from all analyses. Next, we analyzed the prevalence and randomness of missing data among the remaining participants. As the results showed that missing data are rare (none of the items had a response rate of less than 98.9\%) and likely missing completely at random (as shown by Little's Missing Completely at Random test; $\left.\chi^{2}(5587)=5213.63, p=1.000\right)$, we replaced missing values using the expectation-maximization algorithm. We then performed normality tests, calculated skewness, kurtosis, and checked for outliers that might affect the results. As these analyses did not reveal any issues, we calculated basic descriptive statistics and correlations between variables.

Following these steps, we used LPA with maximum likelihood with robust standard errors (MLR) as the estimator to identify subgroups of individuals characterized by similarities in their scientific knowledge, trust in science, and health literacy. The best-fitting profile solution was determined using multiple criteria. First, relative fit information criteria, specifically Akaike Information Criterion (AIC), Bayesian Information Criterion (BIC), and Sample-Adjusted BIC (SABIC) were considered. In these cases, low values indicate a better fit. Second, we took into account the confidence with which individuals have been classified as belonging to one group or another. A standard parameter used for this is entropy, which has a conventional cut-off point of .80 or higher (Clark \& Muthén, 2009). Third, we quantified specific comparisons between the model of interest and a model with one fewer class using the Bootstrapped Likelihood Ratio Test (BLRT). When the result is not significant for a model with $\mathrm{k}+1$ profiles, the solution is not superior to a k-profile solution. Fourth, parsimony and meaningfulness were also considered. For example, some sources report that when the profile includes $<1.0 \%$ of the total 
sample size, the profile should be rejected (Lubke \& Neale, 2006). Lastly, theoretical plausibility, which should be given priority when the fit values allow for this, was also considered (Ram \& Grimm, 2009; Spurk et al., 2020).

To explore differences between the identified profiles, we performed a Pearson's chi-squared test and several analysis of variance (ANOVA) tests. In cases when the data had unequal variances, we performed Welch's ANOVA instead. Significant omnibus tests were followed up with post-hoc tests, using Hochberg's GT2 correction (due to considerably different group sizes) or Games-Howell correction (when the data exhibited unequal variances) to adjust for multiple testing. Given the observed differences in demographic and psychological factors between the profiles, we also used multinomial logistic regression to identify the key predictors of group membership.

\section{Results}

\subsection{Descriptive statistics and correlations}

Basic descriptive statistics ( $M$ and $S D$ ) and correlations between variables are presented in Table 1 below. The results show that scientific knowledge, trust in science, and health literacy are generally weakly positively correlated; the only exception is the correlation between scientific knowledge and health literacy, which is very close to zero. Furthermore, scientific knowledge correlates significantly positively with education and COVID-19 vaccination intention (weak correlations), while it is significantly negatively related to religiosity and conspiracy ideation (weak correlations). Correlations between general scientific knowledge and political conservatism, a health-promoting lifestyle, and COVID-19 compliance did not reach the conventional statistical significance threshold. Trust in science,

on the other hand, is significantly positively correlated with education level, a health-promoting lifestyle, COVID-19 compliance (weak correlations), and COVID-19 vaccination intention (moderate correlation). At the same time, it is significantly negatively correlated with religiosity (weak correlation), political conservatism, and conspiracy ideation (moderate correlations). Lastly, self-reported health literacy is 
associated significantly positively with a health-promoting lifestyle, COVID-19 compliance, and COVID19 vaccination intention (weak correlations). It is also significantly negatively associated with political conservatism and conspiracy ideation (negligible to weak correlations), while its' associations with education and religiosity are not significant. Correlations between the core three constructs (scientific knowledge, trust in science, health literacy) and the HPLP II subscales (nutrition, health responsibility, physical activity, stress management) can be found in the Appendix (Table 7).

[Insert Table 1 about here]

\subsection{Identification and interpretation of profiles}

We investigated various fit statistics for solutions with two to six profiles (Table 2). While relative fit information criteria, entropy, and BLRT generally favor the five-profiles solution, this solution results in two small profiles (1.7\% and $1.1 \%$ of the sample) with limited interpretability. Hence, we chose a more parsimonious and meaningful four-profiles solution. Among the solutions with significant BLRT values, this solution had the second-lowest AIC, BIC, and SABIC. Additionally, the entropy value is above the conventional cut-off and suggests that participants can be allocated to the correct latent profile with sufficient certainty.

[Insert Table 2 about here]

Of particular importance, the four-profiles solution showed four quantitatively different profiles regarding their content (Table 3). Specifically, all three ANOVA tests showed significant differences between the extracted profiles. In the case of scientific knowledge, most post-hoc comparisons were significant at the $p<.001$ threshold. The difference between the first and the second profile was significant at the $p<.050$ level, while comparisons of the second and third profile and the second and fourth profile did not reveal significant differences. In the case of trust in science, all pairwise comparisons showed significant differences $(p<.001)$, while health literacy differed significantly between the first and third profile, first and fourth profile, as well as third and fourth profile $(p<.001)$. 
The remaining pairwise comparisons did not yield significant results, although the comparison between the second and fourth profile also approached significance $(p=.071)$.

\section{[Insert Table 3 about here]}

The first extracted group, which was medium to large in size (Profile $1 ; n=251,35.6 \%$ ), showed low levels in all three indicators, suggesting a poor understanding of science and health information and low trust in scientists and medical experts. The second extracted group, which was small in size (Profile $2 ; n=18,2.6 \%)$, exhibited varied levels in the three indicators. In particular, this group exhibited a moderate comprehension of science in general but very low trust in science and low self-reported health literacy. Next, the third extracted group, which was large in size (Profile 3; $n=328,46.5 \%$ ), exhibited moderate levels in all three indicators. This profile can thus be interpreted as having a moderate understanding of science and medical information and moderate trust in scientists and medical experts. Lastly, the fourth extracted group, which was medium in size (Profile $4 ; n=108,15.3 \%$ ), showed high levels in all three indicators, implying a high understanding of science and medical information as well as high trust in scientists and medical experts. Figure 1 shows the standardized values (below and above the mean) of the indicator variables separately for each extracted profile.

[Insert Figure 1 about here]

\subsection{Differences between the extracted profiles and predictors of profile membership}

There were no differences between the extracted profiles in age $(F(3,701)=1.30, p=.273)$ nor gender $\left(\chi^{2}(6)=10.67, p=.099\right)$. More importantly, we also compared differences across groups in HPB (Table 4) and variables that are traditionally understood as predictors of the indicators used in the present study (Table 5).

[Insert Table 4 about here] 
Due to the violation of the homogeneity of variances assumption, we performed Welch's ANOVA tests for all the variables presented in Table 4. As shown in the table, the four extracted profiles differed significantly in all of the studied health-related outcomes. The post-hoc tests additionally revealed that the health-promoting lifestyle differs significantly between profiles 1 and $3(p=.013)$, profiles 1 and $4(p<.001)$, as well as profiles 3 and $4(p=.009)$, while the comparison between profiles 2 and 4 approached significance $(p=.077)$. The remaining comparisons were not significant. Differences between profiles were even more pronounced in the case of COVID-19 compliance (i.e., all comparisons except the comparison between profiles 1 and 2 were significant) and COVID-19 vaccination intention (all pairwise comparisons yielded significant results). Pairwise comparisons for the HPLP II subscales are presented in the Appendix.

\section{[Insert Table 5 about here]}

Due to the violation of the homogeneity of variances assumption, we again performed Welch's ANOVA tests for all the variables presented in Table 5 and found that the four extracted profiles differ significantly in other variables as well. Pairwise comparisons of education level revealed significant differences between profiles 1 and $3(p=.040)$ and profiles 1 and $4(p<.001)$, while the comparison between profiles 3 and 4 approached significance $(p=.051)$. When comparing religiosity between profiles, only the comparisons between profiles 1 and $4(p<.001)$ and profiles 3 and $4(p=.001)$ yielded significant results. Post-hoc tests for political conservatism mostly led to significant results; the only exceptions are comparisons between profiles 1 and 2 as well as profiles 2 and 3. Lastly, all pairwise comparisons of conspiracy ideation were significant.

The variables presented in Table 5 were also used as predictors of profile membership in a multinomial logistic model. The results showed that the model containing the predictor variables outperforms the model containing only the intercept $\left(\chi^{2}(12)=196.96\right.$, Nagelkerke $\left.R^{2}=.27, p<.001\right)$. A more detailed look reveals that education $\left(\chi^{2}(3)=14.64, p=.002\right)$, religiosity $\left(\chi^{2}(3)=7.87, p=.049\right)$, 
political conservatism $\left(\chi^{2}(3)=38.04, p=<.001\right)$, and conspiracy ideation $\left(\chi^{2}(3)=92.40, p=<.001\right)$ are all significant contributors to the model. The key parameter estimates are reported in Table 6 below.

\section{[Insert Table 6 about here]}

\section{Discussion}

This study aimed to investigate associations between general scientific knowledge, trust in science, and health literacy, determine how these variables are related to HPB, and examine these variables from a person-centered perspective.

While the indicators used were generally weakly positively associated, we, interestingly, observed only a negligible correlation between scientific knowledge and health literacy. This finding might seem surprising at first, as health education and science education are somewhat interconnected and both include some form of knowledge. However, health literacy is more content-specific and includes many additional elements (e.g., skills to find and use health information; Ploomipuu et al., 2019). Moreover, scientific knowledge is conventionally measured with objective tests (Roos, 2014), while health literacy instruments vary in their approach and design, with many of the widely used instruments capturing self-reported, subjective health literacy (Sorensen et al., 2013). These different approaches to measuring the two constructs could further contribute to a low correlation coefficient between the variables.

Furthermore, the results revealed that trust in science and health literacy are positively related to all of the included HPB, while scientific knowledge is only significantly associated with COVID-19 vaccination intention. These results suggest that both trust in science and health literacy are related to HPB in a domain-general way, extending the existing literature on the relationship between trust in science and COVID-19 related health outcomes (Pagliaro et al., 2021; Plohl \& Musil, 2021) and further outlining the importance of fostering health literacy among the public (Nutbeam, 2000). On the other hand, scientific knowledge seems to be more domain-specific. According to our results, it is significantly 
associated with COVID-19 vaccination intention (even more so than health literacy) but shows only a negligible relationship with other HPB. Further research is needed to identify health decisions in which general scientific knowledge is indeed beneficial.

Of particular importance, the findings we present suggest that individuals can be classified into distinct profiles based on their level of trust in science, scientific knowledge, and health literacy. Although some of the parameters involved in deciding on the number of profiles suggested a solution with five profiles, two of these profiles included approximately $1.0 \%$ of the total sample size and were not particularly theoretically meaningful. As suggested by other authors, the theoretically best-fitting solution with acceptable fit values was given priority instead (Spurk et al., 2020). Hence, the final solution consists of four subgroups, which differ in the levels of indicators used, HPB as well as other variables.

In particular, the largest subgroups show moderate levels of all three indicators and low levels of all three indicators, followed by a profile with high levels of all three indicators, and the smallest profile, which exhibited moderate knowledge of science but low self-reported health literacy and very low trust in science. Among the three uniform profiles (low, moderate, and high levels of all three indicators), the profile of individuals with low scientific knowledge, trust in science, and health literacy exhibits the lowest levels of HPB. This is generally in line with the previous literature, which theoretically or empirically linked the three individual indicators with HPB and other health-related outcomes (Berkman et al., 2011; Plohl \& Musil, 2021; Yacoubian, 2018). Interestingly, the additional varied profile, which is very small in size, is equally or even more (in the case of COVID-19 related variables) at-risk than the uniformly low profile even though these individuals exhibit moderate levels of scientific knowledge. Such results imply that scientific knowledge alone may not be a sufficient driver of health decisions.

Additional support for the validity of the identified profiles can be found in the analyses, comparing the profiles based on their education level, religiosity, political conservatism, and conspiracy ideation. For example, political conservatism, religiousness, and conspiracy thinking are well-known predictors of trust in science (e.g., Rutjens et al., 2018; Rutjens \& Lee, 2020). In line with this, our results 
show that the first two profiles, characterized by low or very low trust in science, consist of individuals who are more religious, politically conservative, and prone to conspiracy ideation compared to the remaining profiles. On the other hand, education level has previously been associated with scientific knowledge (Hornsey et al., 2021). As a result, education level was highest in profiles 2 and 4, which exhibit the highest level of scientific knowledge. These findings are further supported by the regression analyses, which show that all four variables are significant predictors of profile membership and that their predictive value differs between specific comparisons in line with previous research.

\subsection{Limitations}

As the data were collected online, via an online panel, some of the disadvantages of online panels, such as panel bias (i.e., panelists are sometimes very experienced in filling out surveys), apply to our study. However, it should be noted that Prime Panels participants are generally less exposed to classic protocols compared to participants on other similar platforms (Chandler et al., 2019). The study results should still be generalized with caution; while the sample was heterogeneous, it slightly diverges from the overall US population in some aspects. For example, women were somewhat overrepresented in the sample (61.0\% versus $50.7 \%$ in the general population), and so were individuals aged $25-54$ (55.5\% versus $49.7 \%$ in the general adult population), 65 or above $(20.3 \%$ versus $17.5 \%$ in the general adult population; Central Intelligence Agency, 2021) and individuals with a college degree $(24.1 \%$ versus $16.8 \%$ in the general population; American National Election Studies, 2016). Additionally, future studies could benefit from a larger sample size; as the smallest profile only includes $2.6 \%$ of the sample, it contains - based on the current sample size - less than 25 cases, which is not ideal for LPA (Lubke \& Neale, 2006). Sample sizes of approximately 1000 participants would thus be needed to fulfill this criterion.

Besides limitations related to sampling, the study's disadvantage is its' cross-sectional nature, making it impossible to form causal inferences. Such design may also contribute to common method bias; as all data were collected at the same time and using the same method (i.e., self-report), the relationships 
between variables may, to some degree, be artificially inflated. Future studies could hence test the observed associations in a longitudinal setting or using objective data (e.g., physical activity data collected by smartphone apps). Lastly, it is worth noting that the present study did not account for all social cognition variables linked to the measured outcomes, such as social norms. Therefore, more studies with additional variables that might explain some of the relationships and differences observed in this study are needed.

\subsection{Conclusion}

Although our study had limitations, we were able to distinguish between four subgroups of individuals based on their scientific knowledge, trust in science, and health literacy. We found three profiles with uniformly low, moderate, and high levels of these variables and a varied profile that exhibits a moderate level of scientific knowledge but low to very low levels of the remaining two variables. The results consistently show that the varied profile and the uniformly low profile exhibit lower levels of various HPB compared to the remaining profiles. While these results are interesting in a descriptive, theoretical sense, they also offer some practical implications for designing public health campaigns. First, they point to specific subgroups that should specifically be targeted with efforts encouraging HPB (i.e., those with low levels of all three variables and those with low levels of trust in science and health literacy but moderate scientific literacy). Second, they also offer some guidance on how such efforts could be designed (i.e., in a way that is easy to comprehend and, in particular, fosters trust in science and scientists).

\section{Acknowledgments}

None.

\section{Disclosure of interest}

The authors report no conflict of interest. 


\section{Data availability statement}

The data that support the findings of this study are available from the corresponding author upon reasonable request. 


\section{References}

Allum, N., Sibley, E., Sturgis, P., \& Stoneman, P. (2014). Religious beliefs, knowledge about science and attitudes towards medical genetics. Public Understanding of Science, 23(7), 833-849. https://doi.org/10.1177/0963662513492485

American National Election Studies. (2016). American National Election Study: 2016 Pilot Study (ICPSR 36390). https://www.icpsr.umich.edu/web/ICPSR/studies/36390/versions/V1

Berkman, N. D., Sheridan, S. L., Donahue, K. E., Halpern, D. J., \& Crotty, K. (2011). Low health literacy and health outcomes: An updated systematic review. Annals of Internal Medicine, 155(2), 97-107. https://doi.org/10.1059/0003-4819-155-2-201107190-00005

Berlin, J. A., \& Colditz, G. A. (1990). A meta-analysis of physical activity in the prevention of coronary heart disease. American Journal of Epidemiology, 132(4), 612-628.

https://doi.org/10.1093/oxfordjournals.aje.a115704

Bode, A. M., \& Dong, Z. (2009). Cancer prevention research - Then and now. Nature Reviews Cancer, 9(7), 508-516. https://doi.org/10.1038/nrc2646.Cancer

Brotherton, R., French, C. C., \& Pickering, A. D. (2013). Measuring belief in conspiracy theories: The generic conspiracist beliefs scale. Frontiers in Psychology, 4, 1-15. https://doi.org/10.3389/fpsyg.2013.00279

Centers for Disease Control and Prevention. (2012). CDC National Health Report Highlights. https://www.cdc.gov/healthreport/publications/compendium.pdf

Central Intelligence Agency. (2021). North America - United States. https://www.cia.gov/the-worldfactbook/countries/united-states/

Chandler, J., Rosenzweig, C., Moss, A. J., Robinson, J., \& Litman, L. (2019). Online panels in social science research: Expanding sampling methods beyond Mechanical Turk. Behavior Research 
Methods, 51, 2022-2038. https://doi.org/10.3758/s13428-019-01273-7

Clark, S., \& Muthén, B. O. (2009). Relating latent class analysis results to variables not included in the analysis. https://www.statmodel.com/download/relatinglca.pdf

Committee on Science, Engineering, and Public Policy. (2009). On being a scientist. National Academy Press.

Dauchet, L., Amouyel, P., Hercberg, S., \& Dallongeville, J. (2006). Fruit and vegetable consumption and risk of coronary heart disease: A meta-analysis of cohort studies. Journal of Nutrition, 136(10), 2588-2593. https://doi.org/10.1093/jn/136.10.2588

Hales, C. M., Carroll, M. D., Fryar, C. D., \& Ogden, C. L. (2020). Prevalence of obesity and severe obesity among adults: United States, 2017-2018. NCHS Data Brief, no 360. National Center for Health Statistics.

Holbrook, J., \& Rannikkmae, M. (2009). The meaning of scientific literacy. International Journal of Environmental and Science Education, 4(3), 257-288. https://doi.org/10.4324/9781003056584-3

Hornsey, M. J., Edwards, M., Lobera, J., Díaz-catalán, C., \& Barlow, F. (2021). Resolving the small pockets problem clarifies the role of education and political ideology in shaping our understanding of vaccine skepticism. British Journal of Psychology. https://doi.org/10.1111/bjop.12500

Impey, C., Buxner, S., Antonellis, J., Johnson, E., \& King, C. (2011). A twenty-year survey of science literacy among college undergraduates. Journal of College Science Teaching, 40(4), 31-37.

Johnson, D. R., Scheitle, C. P., \& Ecklund, E. H. (2015). Individual religiosity and orientation towards science: Reformulating relationships. Sociological Science, 2, 106-124. https://doi.org/10.15195/v2.a7

Khubchandani, J., Sharma, S., Price, J. H., Wiblishauser, M. J., Sharma, M., \& Webb, F. J. (2021). COVID-19 vaccination hesitancy in the United States: A rapid national assessment. Journal of 
Community Health, 46(2), 270-277. https://doi.org/10.1007/s10900-020-00958-x

Kim, J. H., Marks, F., \& Clemens, J. D. (2021). Looking beyond COVID-19 vaccine phase 3 trials. Nature Medicine, 27, 205-211. https://doi.org/10.1038/s41591-021-01230-y

Kivimaki, M., Virtanen, M., Elovainio, M., Kouvonen, A., Vaananen, A., \& Vahtera, J. (2006). Work stress in the etiology of coronary heart disease - A meta-analysis. Scandinavian Journal of Work, Environment, and Health, 32(6), 431-442. https://doi.org/10.5271/sjweh.1049

Laugksch, R. C. (2000). Scientific literacy: A conceptual overview. Science Education, 84(1), 71-94. https://doi.org/10.1002/(SICI)1098-237X(200001)84:1<71::AID-SCE6>3.0.CO;2-C

Law, M. R., Wald, N. J., \& Thompson, S. G. (1994). By how much and how quickly does reduction in serum cholesterol concentration lower risk of ischaemic heart disease? The British Medical Journal, 308, 367-372. https://doi.org/10.1136/bmj.308.6925.367

Lotfi, M., Hamblin, M. R., \& Rezaei, N. (2020). COVID-19: Transmission, prevention, and potential therapeutic opportunities. Clinica Chimica Acta, 508, 254-266. https://doi.org/10.1016/j.cca.2020.05.044

Lubke, G., \& Neale, M. C. (2006). Distinguishing between latent classes and continuous factors: Resolution by maximum likelihood? Multivariate Behavioral Research, 41(4), 499-532. https://doi.org/10.1207/s15327906mbr4104_4

MacDonald, N. E., \& the SAGE Working Group on Vaccine Hesitancy. (2015). Vaccine hesitancy: Definition, scope and determinants. Vaccine, 33, 4161-4164. https://doi.org/10.1016/j.vaccine.2015.04.036

Maniaci, M. R., \& Rogge, R. D. (2014). Caring about carelessness: Participant inattention and its effects on research. Journal of Research in Personality, 48, 61-83. https://doi.org/10.1016/j.jrp.2013.09.008 
McPhetres, J., \& Zuckerman, M. (2018). Religiosity predicts negative attitudes towards science and lower levels of science literacy. PLoS ONE, 13(11), 1-20. https://doi.org/10.1371/journal.pone.0207125

Nadelson, L., Jorcyk, C., Yang, D., Jarratt Smith, M., Matson, S., Cornell, K., \& Husting, V. (2014). I just don't trust them: The development and validation of an assessment instrument to measure trust in science and scientists. School Science and Mathematics, 114(2), 76-86. https://doi.org/10.1111/ssm.12051

Nutbeam, D. (2000). Health literacy as a public health goal: A challenge for contemporary health education and communication strategies into the 21 st century. Health Promotion International, 15(3), 259-267. https://doi.org/10.1093/heapro/15.3.259

Nylund, K. L., Asparouhov, T., \& Muthen, B. O. (2007). Deciding on the number of classes in latent class analysis and growth mixture deciding on the number of classes in latent class analysis and growth mixture modeling: A Monte Carlo simulation study. Structural Equation Modeling: A Multidisciplinary Journal, 14(4), 535-569. https://doi.org/10.1080/10705510701575396

Pagliaro, S., Sacchi, S., Pacilli, M. G., Brambilla, M., Lionetti, F., Bettache, K., Bianchi, M., Biella, M., Bonnot, V., Boza, M., Butera, F., Ceylan-Batur, S., Chong, K., Chopova, T., Crimston, C. R., Alvarez, B., Cuadrado, I., Ellemers, N., Formanowicz, M., ... Zubita, E. (2021). Trust predicts COVID-19 prescribed and discretionary behavioral intentions in 23 countries. PLOS ONE, 16(3), e0248334. https://doi.org/10.1371/journal.pone.0248334

Pelikan, J. M., Ganahl, K., Van den Broucke, S., \& Sorensen, K. (2019). Measuring health literacy in Europe: Introducing the European Health Literacy Survey Questionnaire (HLS-EU-Q). In International handbook of health literacy (pp. 115-138). Policy Press.

Plohl, N., \& Musil, B. (2021). Modeling compliance with COVID-19 prevention guidelines: The critical role of trust in science. Psychology, Health and Medicine, 26(1), 1-12. https://doi.org/10.1080/13548506.2020.1772988 
Ploomipuu, I., Holbrook, J., \& Rannikma, M. (2019). Modelling health literacy on conceptualizations of scientific literacy. Health Promotion International, 35(5), 1210-1219. https://doi.org/10.1093/heapro/daz106

Ram, N., \& Grimm, K. J. (2009). Methods and Measures: Growth mixture modeling: A method for identifying differences in longitudinal change among unobserved groups. International Journal of Behavioral Development, 33(6), 565-576. https://doi.org/10.1177/0165025409343765

Rhodes, R. E., Janssen, I., Bredin, S. S. D., Warburton, D. E. R., \& Bauman, A. (2017). Physical activity: Health impact, prevalence, correlates and interventions. Psychology and Health, 32(8), 942-975. https://doi.org/10.1080/08870446.2017.1325486

Richardson, S., Shaffer, J. A., Falzon, L., Krupka, D., Davidson, K. W., \& Edmondson, D. (2012). Metaanalysis of perceived stress and its association with incident coronary heart disease. American Journal of Cardiology, 110(12), 1711-1716. https://doi.org/10.1016/j.amjcard.2012.08.004

Roos, J. M. (2014). Measuring science or religion? A measurement analysis of the National Science Foundation sponsored science literacy scale 2006-2010. Public Understanding of Science, 23(7), 797-813. https://doi.org/10.1177/0963662512464318

Rutjens, B. T., \& Lee, R. Van Der. (2020). Spiritual skepticism? Heterogeneous science skepticism in the Netherlands. Public Understanding of Science, 29(3), 335-352. https://doi.org/10.1177/0963662520908534

Rutjens, B. T., Sutton, R. M., \& Lee, R. Van Der. (2018). Not all skepticism is equal: Exploring the ideological antecedents of science acceptance and rejection. Personality and Social Psychology Bulletin, 44(3), 384-405. https://doi.org/10.1177/0146167217741314

Sattelmair, J., Pertman, J., Ding, E. L., Kohl, H. W., Haskell, W., \& Lee, I. M. (2011). Dose response between physical activity and risk of coronary heart disease: A meta-analysis. Circulation, 124, 
789-795. https://doi.org/10.1161/CIRCULATIONAHA.110.010710

Sorensen, K., Van den Broucke, S., Pelikan, J. M., Fullam, J., Doyle, G., Slonska, Z., Kondilis, B., Stoffels, V., Osborne, R. H., \& Brand, H. (2013). Measuring health literacy in populations: Illuminating the design and development process of the European Health Literacy Survey Questionnaire (HLS-EU-Q). BMC Public Health, 13, 948. https://doi.org/10.1186/1471-2458-13948

Spurk, D., Hirschi, A., Wang, M., Valero, D., \& Kauffeld, S. (2020). Latent profile analysis: A review and "how to" guide of its application within vocational behavior research. Journal of Vocational Behavior, 120, 103445. https://doi.org/10.1016/j.jvb.2020.103445

Talhelm, T., Haidt, J., Oishi, S., Zhang, X., Miao, F. F., \& Chen, S. (2015). Liberals think more analytically (more "WEIRD”) than conservatives. Personality and Social Psychology Bulletin, 41(2), 250-267. https://doi.org/10.1177/0146167214563672

Von Wagner, C., Knight, K., Steptoe, A., \& Wardle, J. (2007). Functional health literacy and healthpromoting behaviour in a national sample of British adults. Journal of Epidemiology and Community Health, 61(12), 1086-1090. https://doi.org/10.1136/jech.2006.053967

Walker, S. N., \& Hill-Polerecky, D. M. (1996). Psychometric evaluation of the Health-Promoting Lifestyle Profile II. University of Nebraska Medical Center.

World Health Organizaton. (2020). The top 10 causes of death. https://www.who.int/news-room/factsheets/detail/the-top-10-causes-of-death

Yacoubian, H. A. (2018). Scientific literacy for democratic decision-making. International Journal of Science Education, 40(3), 308-327. https://doi.org/10.1080/09500693.2017.1420266 


\title{
Appendix
}

\section{Correlations between trust in science, scientific knowledge, health literacy and HPLP II subscales}

\author{
[Insert Table 7 about here]
}

\section{Pairwise comparisons between profiles: HPLP II subscales}

Results of pairwise comparisons, performed with the Games-Howell post-hoc test, revealed significant differences in nutrition between profiles 1 and $4(p<.001)$ and profiles 1 and $3(p=.012)$, while the difference between profiles 3 and 4 approached significance $(p=.055)$. The remaining pairwise comparisons were not significant.

Similarly, we observed significant differences in health responsibility between profiles 1 and $4(p<.001)$, profiles 1 and $3(p=.019)$, profiles 2 and $4(p=.037)$ and profiles 3 and $4(p=.014)$. The remaining pairwise comparisons were not significant.

Physical activity differed significantly only between profiles 1 and $4(p=.028)$. The remaining pairwise comparisons were not significant.

Lastly, pairwise comparisons revealed that stress management differed significantly between profiles 1 and $4(p<.001)$ and profiles 3 and $4(p=.001)$, while the remaining comparisons did not yield significant results. 


\section{Table 1}

Basic descriptive statistics and correlations between variables

\begin{tabular}{|c|c|c|c|c|c|c|c|c|c|c|}
\hline & $\begin{array}{l}M \\
(S D)\end{array}$ & 1. & 2. & 3. & 4. & 5. & 6. & 7. & 8. & 9. \\
\hline 1. Scientific knowledge & $\begin{array}{l}.55 \\
(.23)\end{array}$ & - & & & & & & & & \\
\hline 2. Trust in science & $\begin{array}{l}3.56 \\
(0.73)\end{array}$ & $.25^{* * * *}$ & - & & & & & & & \\
\hline 3. Health literacy & $\begin{array}{l}3.14 \\
(0.50)\end{array}$ & .04 & $.27^{* * *}$ & - & & & & & & \\
\hline 4. Education & $\begin{array}{l}3.26 \\
(1.02)\end{array}$ & $.22^{* * * *}$ & $.12^{* *}$ & .07 & - & & & & & \\
\hline 5. Religiosity & $\begin{array}{l}3.91 \\
(2.18)\end{array}$ & $-.10^{*}$ & $-.21^{* * * *}$ & .00 & .06 & - & & & & \\
\hline 6. Political conservatism & $\begin{array}{l}3.97 \\
(1.76)\end{array}$ & -.05 & $-.36^{* * *}$ & $-.08^{*}$ & -.02 & $.35^{* * *}$ & - & & & \\
\hline 7. Conspiracy ideation & $\begin{array}{l}2.86 \\
(0.96)\end{array}$ & $-.20^{* * * *}$ & $-.43^{* * * *}$ & $-.11^{* *}$ & $-.10^{* * *}$ & $.08^{*}$ & $.16^{* * * *}$ & - & & \\
\hline 8. Health prom. lifestyle & $\begin{array}{l}2.21 \\
(0.55)\end{array}$ & .06 & $.20^{* * * *}$ & $.25^{* * *}$ & $.27^{* * *}$ & $.17^{* * * *}$ & -.05 & -.02 & - & \\
\hline 9. COVID-19 compliance & $\begin{array}{l}3.43 \\
(0.59)\end{array}$ & .01 & $.28^{* * * *}$ & $.25^{* * *}$ & .01 & -.06 & $-.22^{* * *}$ & $-.17^{* * * *}$ & $.21^{* * *}$ & - \\
\hline 10. COVID-19 vac. intention ${ }^{a}$ & $\begin{array}{l}3.35 \\
(1.60) \\
\end{array}$ & $.20^{* * * *}$ & $.41^{* * * *}$ & $.11^{* *}$ & $.21^{* * * *}$ & -.08 & $-.32^{* * * *}$ & $-.36^{* * * *}$ & $.12^{* *}$ & $.37^{* * * *}$ \\
\hline
\end{tabular}




\section{Table 2}

Fit indices for profile solutions

\begin{tabular}{lllllllll}
\hline Number of profiles & LL & FP & AIC & BIC & SABIC & Entropy & BLRT $(p)$ & Smallest profile size \\
\hline 2 & -1210.31 & 10 & 2440.62 & 2486.21 & 2454.45 & .447 & $<.001^{* * * *}$ & $42.4 \%$ \\
3 & -1181.76 & 14 & 2391.51 & 2455.33 & 2410.87 & .713 & $<.001^{* * * *}$ & $1.1 \%$ \\
4 & -1151.75 & 18 & 2347.50 & 2429.55 & 2372.40 & .825 & $<.001^{* * *}$ & $2.6 \%$ \\
5 & -1137.26 & 22 & 2318.51 & 2418.79 & 2348.94 & .856 & $<.001^{* * *}$ & $1.1 \%$ \\
6 & -1134.23 & 26 & 2320.46 & 2438.97 & 2356.42 & .870 & 1.000 & $0.1 \%$ \\
\hline
\end{tabular}

Notes. ${ }^{*} p<.050,{ }^{* * *} p<.010,{ }^{* * *} p<.001$. 
Table 3

General scientific knowledge, trust in science, and health literacy: profile means

\begin{tabular}{|c|c|c|c|c|c|}
\hline & Profile 1 & Profile 2 & Profile 3 & Profile 4 & \\
\hline & $M(S D)$ & $M(S D)$ & $M(S D)$ & $M(S D)$ & $F$ \\
\hline Scientific knowledge $^{a}$ & $.46(.23)$ & $.60(.20)$ & $.57(.20)$ & $.67(.23)$ & $27.91^{* * * *}$ \\
\hline Trust in science ${ }^{b}$ & $2.92(0.27)$ & $1.60(0.38)$ & $3.80(0.26)$ & $4.68(0.23)$ & $1571.40^{* * * *}$ \\
\hline Health literacy $^{c}$ & $3.00(0.48)$ & $2.92(0.88)$ & $3.15(0.45)$ & $3.48(0.47)$ & $26.64^{* * * *}$ \\
\hline
\end{tabular}


Table 4

Differences between profiles: HPB

\begin{tabular}{|c|c|c|c|c|c|}
\hline & $\begin{array}{l}\text { Profile } 1 \\
M(S D)\end{array}$ & $\begin{array}{l}\text { Profile } 2 \\
M(S D)\end{array}$ & $\begin{array}{l}\text { Profile } 3 \\
M(S D)\end{array}$ & $\begin{array}{l}\text { Profile } 4 \\
M(S D)\end{array}$ & $F$ \\
\hline Health promoting lifestyle $^{\mathrm{a}}$ & $2.10(0.53)$ & $2.06(0.58)$ & $2.23(0.50)$ & $2.44(0.63)$ & $9.06^{* * *}$ \\
\hline Nutrition $^{\mathrm{b}}$ & $2.05(0.60)$ & $2.01(0.73)$ & $2.20(0.57)$ & $2.39(0.68)$ & $7.57^{* * *}$ \\
\hline Health responsibility $^{c}$ & $2.04(0.61)$ & $1.93(0.64)$ & $2.18(0.56)$ & $2.41(0.69)$ & $8.67^{* * * *}$ \\
\hline Physical activity ${ }^{d}$ & $1.98(0.72)$ & $1.94(0.85)$ & $2.10(0.73)$ & $2.26(0.90)$ & $3.10^{*}$ \\
\hline Stress management $\mathrm{e}$ & $2.32(0.62)$ & $2.38(0.59)$ & $2.43(0.57)$ & $2.72(0.71)$ & $8.44^{* * *}$ \\
\hline COVID-19 compliance ${ }^{f}$ & $3.32(0.64)$ & $2.73(1.01)$ & $3.48(0.52)$ & $3.66(0.43)$ & $14.48^{* * *}$ \\
\hline COVID-19 vaccination intention ${ }^{\mathrm{g}}$ & $2.73(1.52)$ & $1.13(0.52)$ & $3.65(1.46)$ & $4.22(1.43)$ & $104.42^{* * *}$ \\
\hline
\end{tabular}




\section{Table 5}

Differences between profiles: other variables

\begin{tabular}{|c|c|c|c|c|c|}
\hline & $\begin{array}{l}\text { Profile } 1 \\
M(S D)\end{array}$ & $\begin{array}{l}\text { Profile } 2 \\
M(S D)\end{array}$ & $\begin{array}{l}\text { Profile } 3 \\
M(S D)\end{array}$ & $\begin{array}{l}\text { Profile } 4 \\
M(S D)\end{array}$ & $F$ \\
\hline Education level $^{\text {a }}$ & $3.07(0.98)$ & $3.50(0.79)$ & $3.30(1.08)$ & $3.56(0.89)$ & $7.97^{* * * *}$ \\
\hline Religiosity $^{\mathrm{b}}$ & $4.30(2.11)$ & $4.39(2.77)$ & $3.91(2.10)$ & $2.95(2.18)$ & $9.78^{* * * *}$ \\
\hline Political conservatism $^{c}$ & $4.50(1.63)$ & $4.89(1.96)$ & $3.89(1.65)$ & $2.86(1.80)$ & $23.44^{* * *}$ \\
\hline Conspiracy ideation $^{\mathrm{e}}$ & $3.18(0.71)$ & $3.80(0.75)$ & $2.80(0.93)$ & $2.12(1.07)$ & $40.06^{* * *}$ \\
\hline
\end{tabular}




\section{Table 6}

Summary of parameter estimates for variables predicting profile membership

\begin{tabular}{|c|c|c|c|}
\hline & $B$ & $S E B$ & Wald \\
\hline \multicolumn{4}{|l|}{ Profile 1} \\
\hline Education & $-0.47^{* *}$ & 0.14 & 11.58 \\
\hline Religiosity & $0.18^{* *}$ & 0.07 & 7.71 \\
\hline Political conservatism & $0.49^{* * * *}$ & 0.09 & 31.99 \\
\hline Conspiracy ideation & $1.18^{* * *}$ & 0.15 & 62.54 \\
\hline \multicolumn{4}{|l|}{ Profile 2} \\
\hline Education & -0.11 & 0.26 & 0.17 \\
\hline Religiosity & 0.16 & 0.13 & 1.50 \\
\hline Political conservatism & $0.61^{* * *}$ & 0.16 & 14.69 \\
\hline Conspiracy ideation & $2.21^{* * *}$ & 0.37 & 36.04 \\
\hline \multicolumn{4}{|l|}{ Profile 3} \\
\hline Education & -0.24 & 0.13 & 3.64 \\
\hline Religiosity & $0.13^{*}$ & 0.06 & 4.48 \\
\hline Political conservatism & $0.30^{* * *}$ & 0.08 & 14.40 \\
\hline Conspiracy ideation & $0.69^{* * *}$ & 0.13 & 28.25 \\
\hline
\end{tabular}




\section{Table 7}

Correlations with HPLP II subscales

\begin{tabular}{|c|c|c|c|c|c|c|c|}
\hline & $\begin{array}{l}M \\
(S D)\end{array}$ & 1. & 2. & 3. & 4. & 5 . & 6. \\
\hline 1. Scientific knowledge & $\begin{array}{l}.55 \\
(.23)\end{array}$ & - & & & & & \\
\hline 2. Trust in science & $\begin{array}{l}3.56 \\
(0.73)\end{array}$ & $.25^{* * * *}$ & - & & & & \\
\hline 3. Health literacy & $\begin{array}{l}3.14 \\
(0.50)\end{array}$ & .04 & $.27^{* * *}$ & - & & & \\
\hline 4. Nutrition & $\begin{array}{l}2.17 \\
(0.61)\end{array}$ & $.09^{*}$ & $.18^{* * *}$ & $.18^{* * *}$ & - & & \\
\hline 5. Health responsibility & $\begin{array}{l}2.16 \\
(0.61)\end{array}$ & -.01 & $.20^{* * *}$ & $.27^{* * *}$ & $.62^{* * *}$ & - & \\
\hline 6. Physical activity & $\begin{array}{l}2.08 \\
(0.76)\end{array}$ & .06 & $.11^{* *}$ & $.14^{* * *}$ & $.62^{* * * *}$ & $.57^{* * *}$ & - \\
\hline 7. Stress management & $\begin{array}{l}2.44 \\
(0.62) \\
\end{array}$ & .06 & $.18^{* * * *}$ & $.24^{* * * *}$ & $.62^{* * *}$ & $.59^{* * *}$ & $.60^{* * * *}$ \\
\hline
\end{tabular}

Notes. ${ }^{*} p<.050,{ }^{* *} p<.010,{ }^{* * *} p<.001$. 


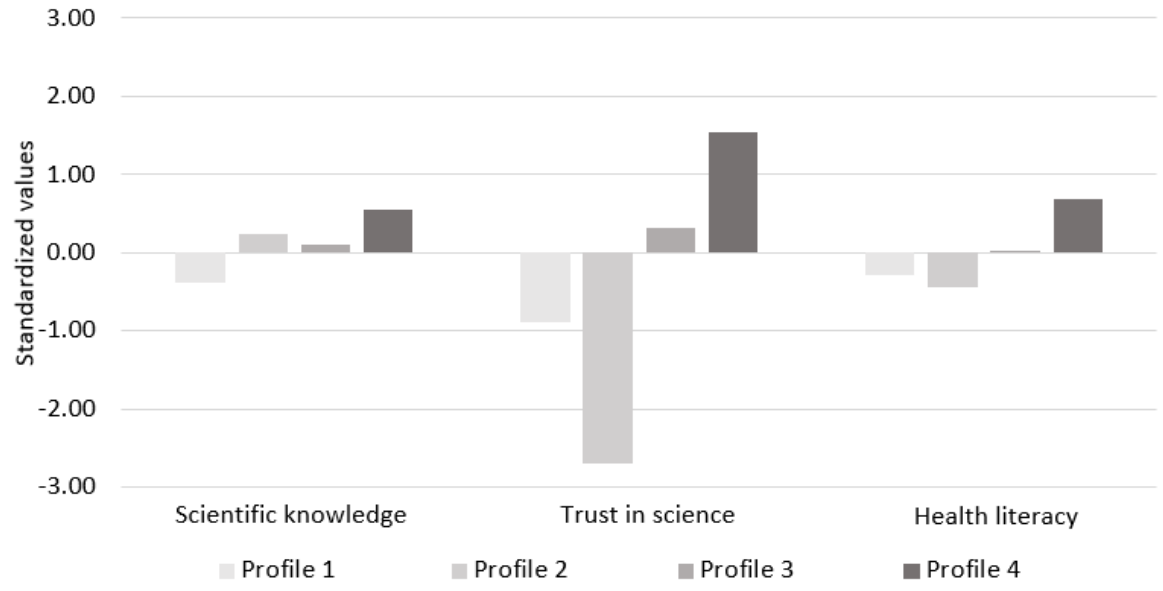

Figure 1. Z-standardized values of the indicator variables. 